\title{
A breast prosthesis infection update: Two-year incidence, risk factors and management at single institution
}

\author{
Ashley Nadia Boustany ${ }^{1}$, Shady Elmaraghi², Nneamaka Agochukwu', \\ Benjamin Cloyd $^{3}$, Adam J. Dugan ${ }^{4}$, Brian Rinker ${ }^{1,3}$ \\ ${ }^{1}$ Department of Surgery, Division of Plastic and Reconstructive Surgery, University of Kentucky, ${ }^{3}$ University of Kentucky School \\ of Medicine, ${ }^{4}$ Department of Biostatistics, University of Kentucky, College of Public Health, Lexington, KY, \\ ${ }^{2}$ Department of Surgery, Division of Plastic and Reconstructive Surgery, University of Wisconsin, Madison, WI, USA
}

Address for correspondence: Dr. Ashley Nadia Boustany, Department of Surgery, Division of Plastic Surgery, University of Kentucky, 740 S. Limestone, Lexington, KY 40536, USA. E-mail: abo242@uky.edu

\section{ABSTRACT}

Background: Infection following augmentation and prosthetic-based breast reconstruction can cause significant physical and psychological distress for patients. It may delay adjuvant therapies and compromise aesthetic outcomes. The aim of this study is to identify modifiable risk factors for infection and identify common bacterial isolates to achieve optimal outcomes for patients. Methods: A retrospective cohort study was performed for patients undergoing implant-based breast reconstruction over a 2-year period. In each case, we documented demographics, co-morbidities, complications and antibiotic use. We reviewed treatments, infectious species cultured where applicable and all outcomes. Results: A total of 292 patients met the inclusion criteria. Fifty-five patients (19\%) developed an infection. Univariate analysis showed a significantly increased infection rate with longer operative times $(P=0.001)$ and use of tissue expanders $(P=0.001)$. Multiple logistic regression analysis confirmed drain use and elevated body mass index (BMI) as risk factors (odds ratio [OR] 2.427 and 1.061, respectively). After controlling for BMI, smoking status and radiation, we found an increased odd of infection with allograft use (OR 1.838) and a decreased odd with skin preparation using $2 \%$ chlorhexidine gluconate in $70 \%$ isopropyl (OR 0.554), though not statistically significant. Forty of 55 patients with infections had cultures, with $62.5 \%$ of isolates being Gram-positive species and $30 \%$ Gram-negative species. The median time to clinical infection was 25 days. Implant salvage with surgical interventions was achieved in $61.5 \%$ of patients. Conclusions: This study identified judicious use of drains and efficiency in the operating room as modifiable risk factors for infections following implant-based breast reconstruction. Prospective trials to analyse techniques for infection prevention are warranted. Implant salvage following infection is a possible end-point in the appropriate patient.

\section{KEY WORDS}

Breast implant infections; implant salvage; infection risk factors; tissue expander infections

\begin{tabular}{|l|l|}
\hline \multicolumn{2}{|c|}{ Access this article online } \\
\hline Quick Response Code: & Website: \\
\hline & www.ijps.org \\
\cline { 2 - 2 } & Dol: \\
\hline
\end{tabular}

This is an open access journal, and articles are distributed under the terms of the Creative Commons Attribution-NonCommercial-ShareAlike 4.0 License, which allows others to remix, tweak, and build upon the work non-commercially, as long as appropriate credit is given and the new creations are licensed under the identical terms.

For reprints contact: reprints@medknow.com

How to cite this article: Boustany AN, Elmaraghi S, Agochukwu N, Cloyd B, Dugan AJ, Rinker B. A breast prosthesis infection update: Two-year incidence, risk factors and management at single institution. Indian J Plast Surg 2018;51:7-14. 


\section{INTRODUCTION}

$\mathrm{O}$ ne in eight women will develop invasive breast cancer over the course of their lifetime. About $35-40 \%$ of those diagnosed annually will be treated with a total mastectomy, and more of these patients are pursuing breast reconstruction in recent years. In 2013 , over 95,000 reconstructive breast procedures were performed, 75,000 of which were expander-implant-based reconstructions. Infections following augmentation and implant-based breast reconstruction cause significant physical and psychological distress for patients. It delays adjuvant therapies and leads to compromise of aesthetic outcomes. Breast implant infections also pose a significant financial burden on the health-care system. Olsen et al. found that infections after breast operations are associated with a cost over $\$ 4,000$ per patient. ${ }^{[1]}$ Implant infection following breast reconstruction is not an uncommon event; rates cited in the literature range from $2.5 \%$ to $16.5 \%$. Implant infection following breast augmentation is much less common with rates of $1 \%-2.5 \% .{ }^{[2-9]}$

Identification and modification of risk factors for infection leads to better counselling for patients and undoubtedly improves outcomes. Previously described risk factors for the development of implant infections following reconstruction include: Elevated body mass index (BMI), use of drains, smoking, medical co-morbidities, the use of acellular dermal matrix (ADM), concurrent procedures, chemotherapy, radiation therapy and immediate reconstruction. However, there is much variability in the literature as to which factors are the most significant. ${ }^{[2-4,8]}$ Historically, the most common bacterial isolates have been staphylococcal species, but there has been a recent rise in Gram-negative infections..$^{[9]} \mathrm{A}$ better understanding of the most common causative species involved allows reconstructive surgeons to approach the treatment of these patients in a rational and evidence-based manner.

The management of implant-associated infection varies depending on severity. Less severe cases can be treated with outpatient oral antibiotics, while more severe cases necessitate inpatient admission and intravenous antibiotics. The most severe cases result in a failure of reconstruction and implant loss. ${ }^{[7]}$ Attempts for reconstructive salvage, defined as the ability to keep an implant after infection, have also become more popular in recent years. ${ }^{[4,8]}$ The purpose of this study is to identify modifiable risk factors for implant infections, identify the most common causative bacterial isolates, and to analyse and compare success rates for both surgical and conservative management strategies. Our overall goal is to devise a rational and evidence-based approach to the treatment of these patients.

\section{METHODS}

This study received approval from the sponsoring institution's Institutional Review Board, a committee which reviews research protocols to ensure ethical research standards and patient safety. Patients were identified by performing a search by Current Procedural Terminology codes for those who underwent prosthesis-based breast reconstruction over a 2-year period at a single institution. The codes included were 19325: Mammoplasty augmentation with implant; 19328: Removal of intact mammary implant; 19357: Breast reconstruction with tissue expander; 19340: Insertion of the breast prosthesis, immediate; 19342: Delayed insertion of breast prosthesis; and 19330: Removal breast prosthesis. Three hundred and twelve patients were identified. After inclusion criteria were applied and procedures confirmed in operative notes, 292 patients were included in the study. The exclusion criteria included patients under the age of 18 years and those in an active state of confinement in a detention system.

To assess patient characteristics and factors that may influence infection rates we documented the following data points demographics and co-morbidities (age, smoking status, BMI, medical history, American Society of Anaesthesiologists (ASA) classification, prior radiation and perioperative chemotherapy); surgical procedures (augmentation vs. reconstruction, immediate vs. delayed reconstruction, use of autograft or allograft, tissue expander vs. implant placement, additional lymph node dissection, operative time, skin prep aration and pocket irrigation); and perioperative protocols (drain use, perioperative use of antibiotics).

For the purpose of this study, we defined infection as any documentation of breast 'cellulitis', 'erythema', with accompanying warmth, swelling, purulent drainage or pain requiring intravenous or oral antibiotic treatment in the outpatient or inpatient setting. We also defined infection as patients with documentation reporting a diagnosis of implant 'infection' requiring outpatient or inpatient antibiotic therapy, as well as patients with culture-positive swabs of the implant pocket during 
a re-operation for dehiscence or mastectomy flap necrosis.

Within the infected cohort, we documented the species of bacteria cultured, inpatient versus outpatient treatment, success or failure of outpatient treatment, and time to infection. In addition, we looked at the concomitant presence of additional complications including implant exposure, seroma, haematoma and wound dehiscence. Operative interventions undertaken to treat infections were recorded. We compared the demographics and outcomes between patients who developed an infection and those who did not.

Nominal categorical variables were compared using Chi-square and Fisher's exact tests, as appropriate. Continuous variables were tested for normality using the Shapiro-Wilk test for normality along with histograms. Normally distributed continuous variables were compared using $t$-tests; otherwise, Mann-Whitney U-tests were used. Univariate odds ratios (ORs) were calculated using logistic regression models. Statistical significance was defined as a $P<0.05$.

A multiple logistic regression model was used to find predictors of infection among the reconstruction cases. A full model was created with main effects for all pre- and peri-operative variables that were found to have univariate $P<0.2$ in their relationship with infection. Then, a backwards elimination procedure was applied where variables were removed one at a time if and only if doing so reduced the model's Akaike's Information Criterion (AIC) since a lower AIC implies a better fit to the data. Prior studies have suggested that smoking status, BMI and radiation exposure may contribute to increased rates of infection. ${ }^{[10,11]}$ To see if graft type and surgical prep had an effect on infection rates independent of these known risk factors, two separate logistic regression models were fit to the data. One model looked at the effect of graft type controlling for smoking status, BMI and radiation exposure, while the other looks at the effect of surgical prep controlling for smoking status, BMI and radiation exposure. Goodness of fit of the logistic regression models was tested using the Hosmer-Lemeshow test. Multicollinearity among the predictors was assessed using generalised variance-inflation factors. The assumption of linearity in the logit was tested for continuous predictors using the Box-Tidwell transformation. All statistical analyses were performed in $\mathrm{R}$ programming language, version 3.4.3 (R Core Team; Vienna, Austria).

\section{RESULTS}

A total of 292 patients were included in the study after the inclusion and exclusion criteria were applied. Fifty-five patients developed an implant infection for an infection rate of $18.8 \%$. All of the infections were in the reconstructive cohort, with a $0 \%$ complication rate in the cosmetic augmentation group (32 cases total). The median time from implant placement to infection was 25 days (range $6-448$ days).

\section{Patient characteristics and risk factors}

Themean agewas 48years (range 18-79years). Older age did not correlate with the development of an implant infection. The mean BMI was $28 \mathrm{~kg} / \mathrm{m}^{2}$ (range 17.7-46.8 kg/m²). Elevated BMI was a statistically significant risk factor for the development on an infection $(P=0.001)$. ASA class, diabetes and smoking status were not found to be statistically significant predictors of infection [Table 1].

Operative duration had a statistically significant impact on the development of an infection, with longer operative times resulting in a higher infection rate $(P=0.021)$. Tissue expanders were more likely to become infected than permanent implants $(P=0.001)$. The timing of reconstruction did not have an impact on the development of an infection. Lymph node dissection was not associated with an increased risk of infection, nor was the use of allograft or autograft, the type of antibiotic used, perioperative chemotherapy or radiation therapy [Table 2]. The type of skin antiseptic used to

Table 1: Patient characteristics and risk factors

\begin{tabular}{lcccc}
\hline Variable & All implants & Infections & Noninfections & $\boldsymbol{P}$ \\
\hline $\begin{array}{l}\text { Number of } \\
\text { procedures (\%) }\end{array}$ & 292 & $55(19)$ & $237(81)$ & \\
Age (years) & $48 \pm 12$ & $49 \pm 12$ & $48 \pm 12$ & 0.577 \\
BMI (kg/m²) & $28 \pm 6.4$ & $31 \pm 6$ & $27 \pm 6$ & 0.001 \\
ASA class (\%) & & & & \\
1 & $26(8.9)$ & $2(3.6)$ & $24(10)$ & 0.059 \\
2 & $184(63)$ & $33(60)$ & $151(64)$ & \\
3 or 4 & $82(28)$ & $20(36)$ & $62(26)$ & \\
Diabetes & $31(11)$ & $6(11)$ & $25(11)$ & 1.000 \\
mellitus (\%) & & & & \\
Cosmetic (\%) & $32(11)$ & 0 & $32(14)$ & 0.008 \\
Operative & $2: 24$ & $3: 31$ & $2: 14$ & 0.021 \\
duration & $(1: 15-4: 36)$ & $(1: 31-5: 07)$ & $(1: 15-4: 15)$ & \\
Smoking & & & & \\
status (\%) & & & & \\
$\quad$ Never & $176(60)$ & $29(53)$ & $147(62)$ & 0.140 \\
Quit & $54(19)$ & $10(18)$ & $44(19)$ & \\
Current & $62(21)$ & $16(29)$ & $46(19)$ & \\
\hline Test for ordina tras & & & &
\end{tabular}

Test for ordinal trend was performed for ASA class and smoking status. ASA: American Society of Anesthesiologists 
prepare the skin and the initial fill volume also did not affect the implant infection rate [Tables 3 and 4]. The use of surgical drains with implant placement did have a statistically significant impact on the development of an infection $(P=0.032)$. Further, increased hospital length of stay led to a statistically significant increase in implant infection rates $(P=0.001)$.

Of the 55 patients, who developed an implant infection, 25 (45.4\%) had an additional complication. These included seromas $(n=7)$, skin flap necrosis $(n=5)$, wound dehiscence with or without implant exposure $(n=12)$, implant leaks $(n=2)$ or a haematoma $(n=1)$. The type of pocket irrigation had no effect on implant infection rates.
A backwards elimination stepwise procedure was used to develop a multiple logistic regression model for predicting infection among reconstructions and found BMI and drain used to be most predictive of infection. In this regression model, we observed that drain use was associated with a 2.4-fold increase (OR 2.427; 95\% confidence interval $[\mathrm{CI}] 1.208,5.252 ; P=0.0171$ ) in the odds of an implant infection and a 1 unit increase in BMI was associated with a $6.3 \%$ increase (OR 1.061; 95\% CI 1.014, 1.114; $P=0.0109$ ) in the odds of an implant infection [Table 5]. Two additional multiple logistic regression models were fit to investigate the relationships between graft type and surgical prep and infection after controlling for BMI, smoking status and

Table 2: Risk factors for infection in the reconstructive cohort

\begin{tabular}{|c|c|c|c|c|}
\hline Variable & All implants & Infections & Noninfections & $\boldsymbol{P}$ \\
\hline Number procedures (\%) & 260 & $55(21)$ & $205(79)$ & \\
\hline \multicolumn{5}{|l|}{ Side $(\%)$} \\
\hline Left only & $46(18)$ & $6(11)$ & $40(20)$ & \multirow[t]{3}{*}{0.325} \\
\hline Right only & $56(22)$ & $13(24)$ & $43(21)$ & \\
\hline Bilateral & $157(61)$ & $36(66)$ & $121(59)$ & \\
\hline \multicolumn{5}{|l|}{ Implant type (\%) } \\
\hline Permanent & $104(40)$ & $14(26)$ & $90(44)$ & \multirow[t]{2}{*}{0.020} \\
\hline Tissue expander & $156(60)$ & $41(74)$ & $115(56)$ & \\
\hline Delayed timing & $49(19)$ & $7(13)$ & $42(21)$ & 0.224 \\
\hline Lymph node dissection & $30(12)$ & $4(7.5)$ & $26(13)$ & 0.400 \\
\hline \multicolumn{5}{|l|}{ Use of graft $(\%)$} \\
\hline Allograft & $132(51)$ & $29(53)$ & $103(50)$ & \multirow[t]{3}{*}{0.124} \\
\hline Autograft & $56(22)$ & $16(29)$ & $40(20)$ & \\
\hline Neither & $72(28)$ & $10(18)$ & $62(30)$ & \\
\hline Drain(s) & $173(67)$ & $44(80)$ & $129(63)$ & 0.026 \\
\hline \multicolumn{5}{|l|}{ Intraoperative antibiotic (\%) } \\
\hline Cefazolin & $208(80)$ & $45(82)$ & $163(80)$ & \multirow[t]{3}{*}{0.203} \\
\hline Clindamycin & $41(16)$ & $6(11)$ & $35(17)$ & \\
\hline Vancomycin, other & $10(3.9)$ & $4(7.3)$ & $6(2.9)$ & \\
\hline \multicolumn{5}{|l|}{ Chemotherapy (\%) } \\
\hline None & $156(60)$ & $38(69)$ & $118(58)$ & \multirow[t]{3}{*}{0.265} \\
\hline Presurgery & $87(34)$ & $15(27)$ & $72(35)$ & \\
\hline Postsurgery with implant in place & $17(6.5)$ & $2(3.6)$ & $15(7.3)$ & \\
\hline Any presurgery chemotherapy & $87(34)$ & $15(27)$ & $72(35)$ & 0.350 \\
\hline \multicolumn{5}{|l|}{ Radiation therapy (\%) } \\
\hline None & $220(85)$ & $44(80)$ & $176(86)$ & \multirow[t]{3}{*}{0.297} \\
\hline Presurgery & $32(12)$ & $8(15)$ & $24(12)$ & \\
\hline Postsurgery with implant in place & $7(2.7)$ & $3(5.5)$ & $4(2.0)$ & \\
\hline Any presurgery radiation therapy & $32(12)$ & $8(15)$ & $24(12)$ & 0.745 \\
\hline
\end{tabular}

Table 3: Surgical prep solutions

\begin{tabular}{|c|c|c|c|c|}
\hline Variable & All implants & Infections & Noninfections & $P$ \\
\hline \multicolumn{5}{|l|}{ Surgical prep, $n(\%)$} \\
\hline $4 \%$ chlorhexidine gluconate + isopropyl alcohol & $67(24)$ & $19(35)$ & $48(21)$ & 0.131 \\
\hline Povidone-iodine & $146(52)$ & $23(43)$ & $123(54)$ & \\
\hline $4 \%$ chlorhexidine & $38(13)$ & $9(17)$ & $29(13)$ & \\
\hline $2 \%$ chlorhexidine gluconate in $70 \%$ isopropyl alcohol & $30(11)$ & $3(5.6)$ & $27(12)$ & \\
\hline $2 \%$ chlorhexidine gluconate in $70 \%$ isopropyl alcohol + povidone-iodine & $2(0.7)$ & 0 & $2(0.9)$ & \\
\hline
\end{tabular}


radiation exposure. We observed an increased odds of infection with the use of allograft (OR 1.838), but it did not reach statistical significance $(P=0.1507)$. Regarding surgical prep, there was an increased odds of infection with the use of isopropyl alcohol with $4 \%$ chlorhexidine and $4 \%$ chlorhexidine alone compared to povidone-iodine (OR 2.099 and 1.156, respectively). There was a decreased odds of infection for $2 \%$ chlorhexidine gluconate in $70 \%$ isopropyl alcohol alone compared to povidone-iodine (ORs 0.554). None of these reached statistical significance.

\section{Causative bacteria}

Fifteen patients did not have wound cultures; thus, 40 cultures were analysed. In total, $62.5 \%$ of the isolates were Gram-positives, with $57.5 \%$ being staphylococcal species. Thirty percent were Gram-negatives [Table 6 for details of isolates]. There were three cases with no growth and three with mixed skin flora.

\section{Management of infections}

Outpatient treatment with oral antibiotics was attempted in 40 of the 55 patients who developed an infection. Twenty of forty patients $(50 \%)$ were successfully treated outpatient with complete resolution of their infection without admission or surgical intervention. Thirty-five

Table 4: Patient characteristics and outcomes among patients with infections

\begin{tabular}{lc}
\hline Variable & All infections \\
\hline Median operative duration (IQR), min & $211(91-307)$ \\
Median BMI (IQR) & $29.9(26.8-33.8)$ \\
Tissue expander, $n(\%)$ & $41(74)$ \\
Median fill volume (IQR), ml & $100(100-150)$ \\
Median time to infection (IQR), days & $25(17-39)$ \\
Outpatient treatment with oral antibiotics, $n(\%)$ & \\
Failed & $20(36.4)$ \\
Successful & $20(36.4)$ \\
Not attempted & $15(27.2)$ \\
Treatment, $n$ (\%) & \\
Implant not removed, nonoperative & $20(36.4)$ \\
Implant removed/not replaced in same & $22(40)$ \\
surgery & \\
Removed/new implant placed in same surgery & $11(20)$ \\
Washout, original implant not removed & $2(3.6)$ \\
\hline IQR: Interquartile range, BMl: Body mass index &
\end{tabular}

Table 5: Summary of the reduced multiple logistic regression model for predicting infection among reconstructions ( $n=260$ )

\begin{tabular}{|c|c|c|c|c|c|c|}
\hline \multirow[t]{2}{*}{ Variable } & \multicolumn{3}{|c|}{ Univariate results (controlling for no other variables) } & \multicolumn{3}{|c|}{ Multivariable results* } \\
\hline & OR & $95 \% \mathrm{Cl}$ for the OR & $P$ & OR & $95 \% \mathrm{Cl}$ for the OR & $\boldsymbol{P}$ \\
\hline Intercept & $\mathrm{N} / \mathrm{A}$ & $\mathrm{N} / \mathrm{A}$ & $\mathrm{N} / \mathrm{A}$ & 0.024 & $0.005-0.110$ & $<0.0001$ \\
\hline BMI & 1.061 & 1.013-1.111 & 0.0118 & 1.063 & $1.014-1.114$ & 0.0109 \\
\hline Drain use & 2.375 & $1.193-5.089$ & 0.0184 & 2.427 & $1.208-5.252$ & 0.0171 \\
\hline
\end{tabular}

${ }^{*}$ Multivariable results come from the reduced multiple logistic regression model with BMI and drain use as main effects. OR: Odds ratio, Cl: Confidence interval, N/A: Not available, BMI: Body mass index of the fifty-five patients (63.6\%) who developed an implant infection required an operation. Operative interventions included exploration and pocket lavage without explantation $(n=2)$, implant removal and replacement with a new implant $(n=11)$ and implant removal without replacement $(n=22)$. Twenty patients (36\%) with implant infections were successfully treated non-operatively with antibiotics alone [Table 4].

Implant salvage, or the continued presence of an implant after an operation (not necessarily the same implant) as defined by Nahebedian and Spear, was attempted in 13 patients. This was successful in eight of these 13 patients (61.5\%). Five patients ultimately required implant removal.

\section{DISCUSSION}

The infection rate in the present study was $18.8 \%$, which falls on the high end of the range cited in the literature. However, it should be noted that our definition of infection was fairly broad when compared to that of other studies. For example, Francis et al.'s study on tissue expander infections found a rate of infection of 16.5\%. Their definition of infection was any case where antibiotics were given in response to clinical signs of infection within 1 year from implant placed. ${ }^{[3]}$ In contrast, Cordeiro and McCarthy study of 1521 tissue expanders found a much lower infection rate of $2.5 \%{ }^{[2]}$ However, they defined infection as those patients who were re-admitted to the hospital. Feldman et al. cited an infection rate of $11 \%$, but they limited their definition of infection as that occurring only within the $1^{\text {st }}$ month following surgery. ${ }^{[12]}$

We did not find a statistically significant association between ADM and increased infection rates; however, Chun did find a significantly increased infection rate with ADM use; however, Chun performed a follow-up study showing no difference when two drains were used, and the threshold for drain removal was decreased to $20 \mathrm{ml}$ over 24 h rather than $30 \mathrm{ml}$ over 24 h. ${ }^{[10,13,14]}$ Nahabedian there was a trend towards significance. Weichman and 
Table 6: Isolated species in the infection cohort

\begin{tabular}{lcc} 
Species & $\begin{array}{c}\text { Number of } \\
\text { isolates }\end{array}$ & $\begin{array}{c}\text { Percentage } \\
\text { of total }\end{array}$ \\
\hline MRSA & 6 & 15 \\
MSSA & 9 & 22.5 \\
Staphylococcus epidermidis & 6 & 15 \\
Staphylococcus lugdunensis & 1 & 2.5 \\
Propionibacterium acnes & 1 & 2.5 \\
Corynebacterium striatum & 1 & 2.5 \\
CoNS & 1 & 2.5 \\
No growth & 3 & 7.5 \\
Mixed skin flora & 3 & 7.5 \\
Pseudomonas aeruginosa & 4 & 10 \\
Enterobacter cloacae & 3 & 7.5 \\
Proteus mirabilis & 3 & 7.5 \\
Acinetobacter Iwoffii & 1 & 2.5 \\
Serratia marcescens & 1 & 2.5
\end{tabular}

There were no cultures for 15 patients. MRSA: Methicillin-resistant Staphylococcus aureus, MSSA: Methicillin sensitive Staphylococcus aureus, CoNS: Coagulase-negative Staphylococcus

and Reish did not show a statistically significant association with ADM use. ${ }^{[4,7]}$ The use of dermal autograft also did not result in a significantly increased infection rate, which is in concordance with previous studies. ${ }^{[15,16]}$

We found decreased odds of infection with skin preparation using $2 \%$ chlorhexidine gluconate in $70 \%$ isopropyl alcohol compared to iodine, although this difference was not statistically significant. A Cochrane review comparing the impact of surgical prep in clean surgery on surgical site infections (SSIs) found no difference in 12 studies. However, in this same review, one study was identified that showed a reduced risk of SSI with the use of a prep consisting of $0.5 \%$ chlorhexidine in alcohol. ${ }^{[17]}$ It should be noted that none of these studies looked specifically at breast prosthesis cases. Breast surgeries are typically categorised as clean-contaminated due to bacterial colonisation of the nipple-areola complex. ${ }^{[18]} \mathrm{A}$ randomised controlled trial by Darouiche et al. of clean-contaminated operations found a chlorhexidine-alcohol prep to be superior to iodine prep in the prevention of infection. ${ }^{[19]}$ Carefully designed randomised controlled trials are needed to definitively determine the ideal surgical prep for breast surgery.

Multiple studies have evaluated the impact of irrigation of the breast pocket with various antibiotic solutions. Most of these studies focused on the development of capsular contracture, as the broadly accepted etiology of capsular contracture is a subclinical infection and the formation of biofilms ${ }^{[20-23]}$. Adams et al. found that triple antibiotic irrigation with iodine, cefazolin and gentamicin reduced rates of peri-prosthetic capsular contracture. In a similar study these authors found that a triple antibiotic irrigation mixture consisting of bacitracin, cefazolin and gentamicin was an equivalent alternative. ${ }^{[20,21]}$ In our study, we found no difference in infection rates with single antibiotic pocket irrigation versus saline alone. However, these prior studies indicate that incorporation of a triple antibiotic technique may be beneficial.

The association of implant infection and longer operative times may be related to the duration of implant or pocket exposure to potential contaminants. These contaminants could originate from accidental non-sterile contact with surgeons or circulating staff, surgical instruments or irrigation or circulating air through the ventilation system. We suspect that the longer the wound is open to the air, the more opportunities there are for contamination. More attention to timely closure of the incision is warranted. An area of interest is the effect of limiting the flow of personnel in and out of the operating room while the implant is exposed.

We found a significant correlation between the use of drains and infection. Prior studies have shown a decrease in breast implant infections with continued use of oral antibiotic prophylaxis until drain removal. ${ }^{[24]}$ However, there is no consensus in the literature. ${ }^{[25]}$ The Surgical Care Improvement Project guidelines recommend discontinuation of antibiotics after 24 h. However, breast reconstruction patients may benefit from an extended course of antibiotics due to the marginal blood flow to post-mastectomy skin flaps, presence of a breast prosthesis and the inherent bacterial flora of the nipple-areola complex. ${ }^{[24]}$ The use of prophylactic antibiotics may be warranted in the post-operative period while drains are in place; however, this warrants further study. There is research to suggest that strict adherence to drain care protocols may decrease drain colonisation and subsequent infection. These measures include timely removal of drains, irrigation of the bulb with Dakin's solution, topical mupirocin use, use of chlorhexidine discs and subcutaneous tunnelling of the drain. ${ }^{[26,27]}$ Our current drain removal protocol (removal when the output is $<30 \mathrm{ml} /$ day for 2 consecutive days, not continuing prophylactic antibiotics until drain removal, and the lack of local wound care to drain sites) may contribute to the association with implant infection. The present study emphasises the importance of implementing a comprehensive drain care regimen. 
In our study, the primary bacterial isolates were Staphylococcus species (57.5\%) and Gram-negative rods (30\%). Of the staphylococcal isolates, $20 \%$ were coagulase-negative Staphylococcus (CoNS), 15\% were methicillin-resistant Staphylococcus aureus (MRSA) and $22.5 \%$ were methicillin-sensitive $S$. aureus (MSSA). These findings were consistent with Cohen et al.'s study that found a very similar distribution (27\% CoNS, $7 \%$ MRSA, 25\% MSSA and 20\% Gram-negative species). In contrast, Feldman's study of 31 infections found a higher concentration of MRSA (45\%) and fewer Gram-negative isolates (6\%). These findings indicate the importance of understanding our local microbiomes to target antibiotic treatment appropriately. ${ }^{[9]}$ The rising prevalence of MRSA and Gram-negative species in more recent studies justifies the initial use of broad antibiotic coverage when treating a breast implant infection until microbiological speciation and subsequent antibiotic de-escalation can occur. $^{[9,10]}$

Multiple studies have demonstrated general resistance of many bacterial species to the commonly used first generation cephalosporins in the perioperative period. Several authors suggest that oral antibiotic prophylaxis be with trimethoprim/sulfamethoxazole (or fluoroquinolones) and in the case of a breast implant infections broad empiric treatment with vancomycin, daptomycin or rifampin given the evolution of regional antibiograms. ${ }^{[10,11]}$ Further research is warranted to determine the most effective antibiotic regimen, but a transition to prophylactic trimethoprim/sulfamethoxazole in implant patients is being strongly considered.

Our implant salvage rate was found to be $61.5 \%$. This is comparable to Spear and Seruya whose salvage rate was $64 \% \cdot{ }^{17,8]}$ In the study by Reish $\mathrm{et}$ al. where the implant salvage rate was found to be $37.3 \%$, higher white blood cell counts and MRSA isolates were associated with lower salvage success rates. ${ }^{[7]}$ Lower rates of MRSA in our study may be responsible for the higher success rate of implant salvage. We did not specifically look at predictors for implant salvage in our population, but this is an area of focus moving forwards.

\section{CONCLUSIONS}

The rate of breast implant infections at our institution and in the surgical literature is exceedingly high, emphasising the significance of this problem. Thus, it is important to explore risk factors, interventions and ideal treatment regimens to better address and reduce the incidence of infection. Our study is unique in that it includes a broader definition of infection, involves a complex patient population with more co-morbidities, and provides an updated analysis over the past 2 years. In our study, statistically significant factors for implant infection include elevated BMI, use of tissue expanders, increased operative times and the use of drains. More studies are warranted to further investigate antibiotic regimens and methods to improve implant salvage.

\section{Financial support and sponsorship}

Nil.

\section{Conflicts of interest}

There are no conflicts of interest.

\section{REFERENCES}

1. Olsen MA, Chu-Ongsakul S, Brandt KE, Dietz JR, Mayfield J, Fraser VJ, et al. Hospital-associated costs due to surgical site infection after breast surgery. Arch Surg 2008;143:53-60.

2. Cordeiro PG, McCarthy CM. A single surgeon's 12-year experience with tissue expander/implant breast reconstruction: Part I. A prospective analysis of early complications. Plast Reconstr Surg 2006;118:825-31.

3. Francis SH, Ruberg RL, Stevenson KB, Beck CE, Ruppert AS, Harper JT, et al. Independent risk factors for infection in tissue expander breast reconstruction. Plast Reconstr Surg 2009;124:1790-6.

4. Nahabedian MY, Tsangaris T, Momen B, Manson PN. Infectious complications following breast reconstruction with expanders and implants. Plast Reconstr Surg 2003;112:467-76.

5. Araco A, Gravante G, Araco F, Delogu D, Cervelli V, Walgenbach $\mathrm{K}$, et al. Infections of breast implants in aesthetic breast augmentations: A single-center review of 3,002 patients. Aesthetic Plast Surg 2007;31:325-9.

6. Gabriel SE, Woods JE, O'Fallon WM, Beard CM, Kurland LT, Melton LJ $3^{\text {rd }}$, et al. Complications leading to surgery after breast implantation. N Engl J Med 1997;336:677-82.

7. Reish RG, Damjanovic B, Austen WG Jr., Winograd J, Liao EC, Cetrulo CL, et al. Infection following implant-based reconstruction in 1952 consecutive breast reconstructions: Salvage rates and predictors of success. Plast Reconstr Surg 2013;131:1223-30.

8. Spear SL, Seruya M. Management of the infected or exposed breast prosthesis: A single surgeon's 15-year experience with 69 patients. Plast Reconstr Surg 2010;125:1074-84.

9. Cohen JB, Carroll C, Tenenbaum MM, Myckatyn TM. Breast implant-associated infections: The role of the national surgical quality improvement program and the local microbiome. Plast Reconstr Surg 2015;136:921-9.

10. Chun YS, Verma K, Rosen H, Lipsitz S, Morris D, Kenney P, et al. Implant-based breast reconstruction using acellular dermal matrix and the risk of postoperative complications. Plast Reconstr Surg 2010;125:429-36.

11. Pinsolle V, Grinfeder C, Mathoulin-Pelissier S, Faucher A. Complications analysis of 266 immediate breast reconstructions. J Plast Reconstr Aesthet Surg 2006;59:1017-24.

12. Feldman EM, Kontoyiannis DP, Sharabi SE, Lee E, Kaufman Y, 
Heller L, et al. Breast implant infections: Is cefazolin enough? Plast Reconstr Surg 2010;126:779-85.

13. Weichman KE, Wilson SC, Weinstein AL, Hazen A, Levine JP, Choi $\mathrm{M}$, et al. The use of acellular dermal matrix in immediate two-stage tissue expander breast reconstruction. Plast Reconstr Surg 2012;129:1049-58.

14. Ganske I, Verma K, Rosen H, Eriksson E, Chun YS. Minimizing complications with the use of acellular dermal matrix for immediate implant-based breast reconstruction. Ann Plast Surg 2013;71:464-70.

15. Mirzabeigi MN, Lee M, Smartt JM Jr., Jandali S, Sonnad SS, Serletti JM, et al. Extended trimethoprim/sulfamethoxazole prophylaxis for implant reconstruction in the previously irradiated chest wall. Plast Reconstr Surg 2012;129:37e-45e.

16. Lynch MP, Chung MT, Rinker BD. Dermal autografts as a substitute for acellular dermal matrices (ADM) in tissue expander breast reconstruction: A prospective comparative study. J Plast Reconstr Aesthet Surg 2013;66:1534-42.

17. Dumville JC, McFarlane E, Edwards P, Lipp A, Holmes A, Liu Z, et al. Preoperative skin antiseptics for preventing surgical wound infections after clean surgery. Cochrane Database Syst Rev 2013;3:CD003949.

18. Kataria K, Bagdia A, Srivastava A. Are breast surgical operations clean or clean contaminated? Indian J Surg 2015;77:1360-2.

19. Darouiche RO, Wall MJ Jr., Itani KM, Otterson MF, Webb AL, Carrick MM, et al. Chlorhexidine-alcohol versus povidone-iodine for surgical-site antisepsis. N Engl J Med 2010;362:18-26.

20. Adams WP Jr., Conner WC, Barton FE Jr., Rohrich RJ. Optimizing breast-pocket irrigation: The post-betadine era. Plast Reconstr Surg 2001;107:1596-601.

21. Adams WP Jr., Rios JL, Smith SJ. Enhancing patient outcomes in aesthetic and reconstructive breast surgery using triple antibiotic breast irrigation: Six-year prospective clinical study. Plast Reconstr Surg 2006;117:30-6.

22. Wiener TC. The role of betadine irrigation in breast augmentation. Plast Reconstr Surg 2007;119:12-5.

23. Yalanis GC, Liu EW, Cheng HT. Efficacy and safety of povidone-iodine irrigation in reducing the risk of capsular contracture in aesthetic breast augmentation: A Systematic review and meta-analysis. Plast Reconstr Surg 2015;136:687-98.

24. Clayton JL, Bazakas A, Lee CN, Hultman CS, Halvorson EG. Once is not enough: Withholding postoperative prophylactic antibiotics in prosthetic breast reconstruction is associated with an increased risk of infection. Plast Reconstr Surg 2012;130:495-502.

25. Phillips BT, Bishawi M, Dagum AB, Khan SU, Bui DT. A systematic review of antibiotic use and infection in breast reconstruction: What is the evidence? Plast Reconstr Surg 2013;131:1-3.

26. Murray JD, Elwood ET, Jones GE, Barrick R, Feng J. Decreasing expander breast infection: A new drain care protocol. Can J Plast Surg 2009;17:17-21.

27. Degnim AC, Scow JS, Hoskin TL, Miller JP, Loprinzi M, Boughey JC, et al. Randomized controlled trial to reduce bacterial colonization of surgical drains after breast and axillary operations. Ann Surg 2013;258:240-7. 